\title{
Raising Awareness for Water Polution based on Game Activities using Internet of Things
}

\author{
Chrysanthi Tziortzioti ${ }^{1,2}$, Giuseppe Andreetti ${ }^{3}$, Lucia Rodinò ${ }^{3}$, Irene Mavrommati ${ }^{1,2}$, \\ Andrea Vitaletti ${ }^{3}$ and Ioannis Chatzigiannakis ${ }^{2,3}$ \\ 1 Hellenic Open University, Greece \\ 2 Computer Technology Institute \& Press, Greece \\ 3 Sapienza University of Rome, Italy \\ E-mails: tziortziodgmail.com, \\ andreetti.13391930studenti.uniromal.it, luciatombegmail.com, \\ mavrommatideap.gr, vitaletti@diag.uniromal.it, \\ ichatz@diag.uniroma1.it
}

\begin{abstract}
Awareness among young people regarding the environment and its resources and comprehension of the various factors that interplay, is key to changing human behaviour towards achieving a sustainable planet. In this paper IoT equipment, utilizing sensors for measuring various parameters of water quality, is used in an educational context targeting at a deeper understanding of the use of natural resources towards the adoption of environmentally friendly behaviours. We here note that the use of water sensors in STEM gameful learning is an area which has not received a lot of attention in the previous years. The IoT water sensing and related scenaria and practices, addressing children via discovery, gamification, and educational activities, are discussed in detail.
\end{abstract}

\section{Introduction}

Raising awareness among young people regarding the environmental resources and supporting a better understanding of the factors at play, is key to changing their behaviour towards achieving a sustainable planet. Moreover, reinforcing the educational community on educating new generations has a multiplier effect for understanding the resources and for reducing our environmental footprint; it is the sustainable behaviour at school or social gaming activities that can impact the home behaviour, as children communicate their newly acquired knowledge to parents.

The work presented here focuses on raising awarenes for water polution since most of the planet is covered in water: about $79 \%$ of the surface of the Earth is covered by water. Water has multiple importance as being a source of life as it provides the ability to produce food, minerals and energy, is a key factor for the renewal of the oxygen we breathe, and the means of transporting goods (trade, energy transfer/information). However, in order to achieve a better understanding of the potential of water, its uses, and at the same time to effectively protect it, a detailed study is required [1].

The approach proposed is to develop a set of educational scenaria that use a series of sensors to measure physical and chemical water parameters. As already observed in section 2, bringing the IoT into large aquatic environments is still very difficult, for this 
reason, the focus assumed here is on surface sampling activities that are more affordable in the context of STEM educational activities. This can serve as the first step towards better understanding aquatic resources.

Via the pedagogical steps of awareness, observation, experimentation and action, children using portable and inexpensive equipment will be able to carry out relevant measurements and submit them to a database. The mathematical and scientific thinking developed in the above process can be exploited in various ways by the online application, or the tutor, in the context of teaching mathematical and scientific skills, not only in the science courses but also in cross-thematic approaches that combining such observations and analyzes the economic, social and other aspects of our effort for clean water, and, in subsequent steps, clean seas.

With a systematic, stepwise research approach as a goal, this paper describes the first steps towards this broader direction. With the initial aim to enable children to get involved in the scientific process, of measuring and understanding aquatic parameters, a set of educational scenaria is described; based on these a game has been devised and implemented, deploying a basic IoT sensor-set for measuring water parameters, with the initial goal to measure sweet water resources in one's hometown.

\section{Related work}

Overall, the play was the most typical feature of childhood and was characterized by great autonomy [2]. Several serious games seeking to cultivate environmental awareness, e.g., "Greenpeace" from [28] or the "2020 Energy Game" [3] from the EnergyBits project. However, these are generic Web-based "games", designed and implemented on top of mostly statically defined scenarios, i.e., they do not take into account real data originating from energy meters; also, they do not utilise unique aspects of modern smartphones like location awareness - information presented is the same no matter where the users are located or do not take into account proximity settings. [25] is an example of a browser-based serious game by Siemens, allowing players to test different energy-efficient scenarios in a city-wide planning game. While such designs provide useful insight, they are not paired with real-world data; we will additionally utilise the real-world data produced by the buildings participating in the project. Another example of an energy-focused game is [15], which is being deployed in cooperation with PG\&E, a major US utility company, also discussed in [26].

Regarding the work presented here, [12] discusses pedagogical aspects of integrating real-life data into playful scenarios and serious games; $[21,14]$ are additional examples of such dimensions, that we intend to use while designing and implementing games. [11] discusses the implementation and evaluation of a platform for a multiplayer pervasive game in public open spaces, that we intend to utilise to support our envisioned activities. An example of such activities is discussed in [10], where a university corridor was metamorphosed into an interactive public space. Together with omnipresent devices such as smartphones, an engaging and meaningful experience is brought to life.

Regarding measuring environmental conditions via an IoT network, most of the works focus on terrestrial applications. Even when offshore infrastructures or vessels are considered, IoT devices are mostly deployed in "dry" surfaces and only some spe- 
cific transducers are actually deployed into the water. The underwater environment is hostile, and consequently, underwater IoT devices are very expensive. If you only consider a reliable water-proof housing for shallow water, it costs at least 2 or 3 order of magnitude more than terrestrial solutions and much more if you consider deep water scenarios. Underwater operations are complex and challenging. As an example, the fast growth of algae or microorganisms can suddenly affect the quality of sensors readings that have to be often cleaned. Underwater communications are still extremely difficult and energy-hungry; RF propagates only a few centimetres and only acoustic or optical communications can be used for longer distances. Due to these reasons, the availability of underwater IoT data is still very limited. One of the few attempts to provide a federation of underwater testbeds for the Internet of Underwater Things is the EU project SUNRISE [22]. While SUNRISE clearly showed us the potential of exploring underwater data, it was not originally conceived for STEM educational activities, and both the complexity of the tools and the costs of the equipment are not yet suitable to be operated by students. Despite these difficulties, there are already some efforts for more affordable tools for underwater investigations $[6,20]$ and is, however, possible to design significant STEM activities (see section 4) that focus on shallow water and/or surface sampling that significantly lower the above-discussed difficulties. Indeed, the focus on the shallow water and/or the sea surface allow us to a) engage students in participatory sampling (i.e. they are directly involved in the sampling procedure at sea), b) deploy relatively simple networking infrastructures capable to deliver the data acquired by possible underwater traducers employing standard wireless technologies (e.g. Lora, GPRS or even WiFi).

In the case reported in this paper, a stepwise approach is assumed, by first attempting to measure qualities of sweet water reserves and water resources that are used every day, such as drinking water.

\section{IoT and Real-world Data in STEM}

The aim of this research is to put users in the loop of monitoring as a first step towards raising awareness, but also for gaining knowledge and skills on how to conduct an experiment, and gather information and data with appropriate methods. The concept of users in the loop of monitoring is central in the area of participatory sensing [8] in which personal mobile phones of users are used to collect relevant data for a number of applications such as urban planning, public health, cultural identity and creative expression, and natural resource management. This approach has been employed by the Cornell Laboratory of Ornithology [7] in a science education project on bird biology, while in [19] the authors describe trials for air quality, water quality and plant disease monitoring. Similarly to the context, of this paper [30] presents a solution combining a deployed and participatory sensing system for environmental monitoring.

In [18] the authors discuss the value of participating to project like these for students, concluding that "Students are gaining deep domain-specific knowledge through their citizen science campaign, as well as broad general STEM knowledge through data-collection best practices, data analysis, scientific methods, and other areas specific to their project" 
The GAIA platform $[5,9,4]$ is among few IoT systems that focus on the educational community. The goal of GAIA is to include the users in the loop of monitoring the energy consumption in the buildings they use daily, thus making the first steps towards raising awareness, connecting the educational activities carried out at schools with their activities at home. A real-world IoT deployment is spread in 3 countries, monitoring in real-time 18 school buildings in terms of electricity consumption and indoor and outdoor environmental conditions. GAIA's IoT devices are either open-design IoT nodes (based on the Arduino popular electronics prototyping platform, see [24]) or off-theshelf products acquired from IoT device manufacturers. The data collected is used as part of a series of education scenarios whose goal is to educate, influence and attempt to transform the energy consumption behaviour of elementary school students. Feedback mechanisms notify the students on current energy consumption at school and in this way assist towards raising awareness regarding the environmental effects of energy spending and promote energy literacy by educating the users. Since the GAIA IoT deployment is multi-site it can motivate identification of energy consumption patterns in different geographic locations, and can also serve as the basis for competitions, promoting energy efficiency awareness and sustainability.

The integrated sources of input are utilized to continuously provide direct feedback, custom-tailored to each particular learner/audience. Direct feedback is provided via real-time displays (RTDs) installed at central locations in the buildings, published on school websites, posted on social media, and also displayed on the users' smartphones and tablets. Direct feedback mechanisms are developed to address the immateriality of energy [23] and make it a visible entity by connecting it to the daily activities of students. Visual analytics are combined with recent advances in IoT sensing and pervasive computing technologies to provide an interactive environment that stimulates behavioural change on a frequent basis.

The energy consumption topic is included in the pedagogical activities of the schools incorporating educational aspects to promote energy literacy, convey information regarding historical data and comparative information with other buildings of similar characteristics. A series of social-networking applications are provided to set communitybased incentives for pro-environmental behavioural change and promote collective consuming of resources. These applications utilize the already established relationship between users of the same school/department to provide community-based initiatives to reduce their overall environmental footprint and increase environment-friendly activities. A series of game-based competitions further engage the students in learning how to improve the energy efficiency, and to encourage them to actually follow the learned practices. Research suggests that competitions can be effective in promoting environmentally responsible behaviour [17]. Historical data collected from the IoT infrastructure allows students to compete with each other on periodic intervals (e.g., per week/month/season) to further motivate eco-friendly behaviours. A combination of direct competition among other groups of similar size, climate zone, socio-economic characteristics and past years against each group's own performance is followed. These competitions encourage spreading the word to larger groups, allowing related persons, such as parents, friends, or neighbours, to participate and also appeal to positive emotions, such as hope and enjoyment, as ways to changing individuals behaviours. 


\section{A Game for Raising Awareness for Water Polution}

A set of new educational activities are proposed targeting preliminary/secondary/high schools and addressing the pedagogical needs of existing curricula in aspects related to the environmental impact of water usage and environment-friendly behaviours. The educational scenarios are based on the principle that authentic problems cannot be faced using discrete disciplines that are taught in schools [13]. Quite often students cannot be involved in the learning process because they cannot be engaged in the context in which the problems are embedded [16,29].

The goal of working on water pollution activities using the Internet of Things is to lead to a STEM approach, so the learning becomes connected, focused, meaningful, and relevant to students. As Sanders argues "the focuses of STEM education should apply knowledge of mathematics, science and engineering, design and conduct experiments, analyze and interpret data, and communicate and corporate with multidisciplinary teams" [27].

The educational activities are complemented by game-based methods that offer rich opportunities for a student to learn through engagement with the water quality measurement activities. A meaningful experience is a key to the success of the educational game. Players should voluntarily and actively engage with the game. This is a problem with a lot of serious games that are too much focused on teaching specific content. Especially with students from 13 - 18 years old, who often dismiss anything that feels like educational content. Games are great at providing systems to experiment with, spaces to explore and meanings to be deducted by the players themselves. Meaning that is inherent in the world, the game mechanics, the systems and the narrative of the game. If the educational content is too much focused on forcing specific text-book style knowledge on the players, they will be put off. The work presented here tries to carefully balance the educational content and the freedom of the player to ensure students will become aware of their energy consumption in an unobtrusive manner.

In the educational scenarios proposed, a series of sensors are used to measure physical and chemical water parameters. Using sensors within a water environment for various aspects of water quality is complex and quite challenging. If one only considers a reliable water-proof housing for shallow water, it costs at least 2 or 3 order of magnitude more than terrestrial solutions and much more if the device needs to be submerged into water, and in particular sea water. Underwater communications are still extremely difficult and energy-hungry; RF propagates only a few centimetres and only acoustic or optical communications can be used for longer distances. The energy cost of underwater communications strongly limits the device lifetime, that is usually in the order of few months at best and requires frequent replacements of the batteries, an annoying, time-consuming and difficult task. These are just a couple of examples that explain the very limited availability of underwater IoT. For this reason, the approach presented here focuses on surface sampling activities that are more affordable in the context of STEM educational activities.

The steps of the pedagogical activities followed are awareness, observation, experimentation and action. School students use portable equipment to carry out relevant measurements that are submitted automatically to a central database. Depending on the teaching needs and priorities, students can collect and analyze the following: 
- current values and any fluctuations of them during the observation period of the activity,

- changing values for longer periods of time, e.g. making comparisons between different times of the day, between months, seasons, or years,

- the variance of the phenomena between different areas.

Scientific skills can be developed out of the combination of such observations and utilisation of the system from tutors of various subjects.

\subsection{Educational scenarios and goals}

Students use sensors to monitor physicochemical parameters in their hydrographic network area such as temperature, turbidity, $\mathrm{pH}$, dissolved oxygen (DO) and conductivity. With the accurate and continuous recording of the parameters, they can describe the current physical, chemical and biological state of the water body. The systematic concentration of values creates a database for water quality conditions which can be used to compare data in the future.

The aim is to design and implement systematic research to identify any water quality problems in the water body. Initially, the students use a map of the area to select the position for sampling measurements. Using appropriate sensors, they record the parameter values, plot graphs with spatial and chronological variations, analyze the data, and end up with conclusions on the quality of water. It is important to compare and contrast the effects of water quality parameters, especially the relationship between temperature-DO, turbidity-DO, and $\mathrm{pH}$-turbidity.

Scenario 1: Observation of Water Temperature as one of the most easily measured parameters of water quality and one of the most important factors in the workings of an aquatic ecosystem. Students are asked to make assumptions about the factors on which water temperature depends, such as depth, the hour of day that the measure has been taken, the season of year, mixing of water due to wind and rain, degree of stratification of the water system, the temperature of water flowing from tributaries, and human actions such as drainage of warm water used for cooling water in industrial plants (thermal pollution).

Students with the help of the instructor, conclude that water temperature affects other water quality parameters such as $\mathrm{DO}$ and $\mathrm{pH}$. For example, students count a difference at DO levels as they take measurements early in the morning when the water is cool and then late in the afternoon when the water temperature has increased. Also, there may be a difference in DO levels at different depths of the water if there is a significant change in temperature with depth. The same conclusion will be reached if they measure DO levels at different times of the year. In spring and summer, the upper layer of water gets a higher temperature and mixing between this surface water and the cooler water below. In autumn the air temperature decreases, the surface water becomes more and colder and its density increases. As the surface water is sinking, mixing of the water column is caused. During winter, temperatures remain fairly constant from upper to lower levels (in shallow systems). Considering the above, we expect the students to conclude that water at higher temperatures dissolve lower DO levels than water at a lower temperature. 
Scenario 2: Observation of Water Acidity-Alkalinity by measuring PH as a measure of the balance of acid and alkaline compounds dissolved in water. Generally, natural water has $\mathrm{pH}$ between 5 and 9 and most aquatic organisms survive in this range, so $\mathrm{pH}$ is a key indicator of water quality. Water with low $\mathrm{pH}$ increases the solubility of nutrients such as phosphates and nitrates.

Scenario 3: Observation of Water Turbidity as another physical parameter that measures the number of suspended particles in the water at a specific time and is an indicator of water quality. It depends on suspended solids, such as phytoplankton, mud, sewage, organic matter and industrial waste. High levels of turbidity occur due to natural causes (soil erosion, sediment transfer by rainwater, seasonal conditions like spring melt) and can be caused by human activities (sewage, land use, pollution).

Students are expected to combine high turbidity values with low DO levels in water and raised temperature. Students are also expected to observe that $\mathrm{pH}$ values are affected by turbidity (e.g., disorganized vegetation produces organic acids that lower the $\mathrm{pH}$ ), bacterial activity, photosynthesis rate, and chemical components that end up in the aquatic system.

Students are expected to conclude that the turbidity value is not generally constant along the water column but depends on the depth, seasonality, water mix caused by the wind, storms and tides, the flow of rivers and pollution from human activities.

Scenario 4: Observation of Dissolved Oxygen as an indication of the presence of organisms, particularly microbes, within the water. These organisms with oxidative reactions metabolize organic substances by consuming for this process the oxygen dissolved in the water. Because oxygen has relatively little water solubility, it is quickly consumed when there is a high organic load resulting in anaerobic conditions. Concentration less than $7 \mathrm{mg} / \mathrm{lt}$ means oxygen deficiency resulting in the non-survival of fish and other aerobic organisms. Physiological values of DO range above $7 \mathrm{mg} / \mathrm{lt}$.

The systemic nature of the measurements, as seen above, can be difficult to handle directly. In order to start addressing the problem of understanding water resources aided by IoT sensors, the proposal described in this paper is to start with a small subset of water sensing, and take this into the context of a gaming activity to familiarise oneself with sensors, taking measurements, and the quality of more accessible water, such as springs and drinking water sources.

\subsection{Game Design}

Sensor data are used in the game, as well as location and time data, for following the condition of water sources. The players have to adopt, follow, record and document the data from different water sources. They have to compare between different water sources, but also between the same water source at different times of the day or of the year. They have to make a hypothesis to explain what they observe and document it, and then support their hypothesis by providing facts and related measurements.

For example, if the PH is high, will the organisms live? Which will live and which will die? Until what value will the system maintain its sustainability? One should also try to give a possible explanation why the change occurs, should be able to discuss with others the various possible reasons, and if possible cross checks other parameters 
to support the given explanation. For example, $\mathrm{PH}$ increase may mean someone has thrown something in the water and has contaminated it. Players need to take decisions on what to do in order to remedy the problem, if they can, and restore the system to the previous state of balance. Differences in turbidity, in PH value, or in temperature may be observed, while the different values may happen due to location, time of the day, time of the year, and human-related activities.

Players take their IoT-water explorer sensor kit that contains: a Turbidity sensor, a PH sensor, a Water Temperature Sensor. What the little explorers then do is the following:

1. They are given a kit for water sensing and an accompanying application.

2. They find a water source in their neighbourhood.

3. They use their mobile phones to pin the source location on google map.

4. They take photos of the area and the water source and add it as extra information about the water source they have adopted.

5. They plunge the sensors (Turbidity, $\mathrm{PH}$, and Temperature) in the source, and record the values.

6. They compare the data they have gathered, with a table of related data that shows ranges of normal values.

7. They do it all over again to collect as much water data as possible and expand their area with more sources (a crowd sensing process for water data).

By doing this exploration they develop certain skills: understanding and describing the problem space, documenting aspects of the problem space, taking measurements, following the steps of a so-called scientific process, etc.

The players can play individually, or as part of teams, which compete in an exploration quest. The more records they take, the more they expand their influence area in the city map. The more data and the more sources they document, the more points they gather, in their quest. Later, in different time of the day, and at different seasons during the year, they may return to the water source and take new measurements with their sensor kit, in the water sources that have been previously measured. A diary can be used to view the sensor data added, but also for taking notes on other related activities.

A game scenario can be the following: A set of friends set out to play a scouts game. The goal is to cover an area, by taking measurements in water sources in as many spots in the map as possible. The journey can, in more complete versions, be specific or thematic: one can select seawater, lakes and rivers, drinking water (taps and springs), or thematic (Archeological journey, park journey, temples route, etc).

From the initial deployment, an issue observed relates of grading in the sensors used in IoT games, and the accuracy of sensor data. This "slack" in measurements also affects the results of appropriateness, in a given acceptable range. For example, $\mathrm{PH}$ water measurements in drinking water should be between 6,8 and 8 . If the measurement is outside this scale in a city tap, there may be an issue regarding the validity of the sensor reading. This may owe to the way the measurement is taken, therefore repeating the measurement is encouraged, or to the sensor itself (the grading of sensors has a slack when compared to a more accurate organ). To remedy this, once a measurement is taken and is seen to be outside of the expected range, the player is given an instant warning message to have the sensor graduation checked and repeat the sampling, in 
order to opt out a measuring error, by taking a second measurement from the same water source. Thus the player learns gradually to make sure that (s)he takes thorough and valid measurements. The players are encouraged (by messages and highlighted map locations) to return to take sensor readings at different time intervals (during the day, week, month, or in different seasons. If other players take measurements they co-own the area, and the rights of the previous players are weakened. Furthermore, they can compare explanations and exchange opinions with tutors and with their groups.

\section{Deployment \& Testing in Real-world Environments}

The design of the IoT system, the educational scenarios and corresponding gamification activities are evaluated to measure the relevance of a realized system with the goals initially identified. The evaluation follows an experimental approach examining the performance of certain technical components through an operation in real-world environments. Moreover, the complete system is to be evaluated from the end-users perspective as an integrated interaction experience.

\subsection{Hardware Prototype}

A hardware prototype device was developed for the realization of the measuring kit provided to the end-users. The measuring kit is developed to offer a simplified interaction with the students that do not require the combination of multiple devices for the storage and transfer of data or for the power. It is expected that through a simplified experience the students will focus on carrying out the gaming elements. For this reason, the device was developed to operate independently from any other device, such as a smartphone or tablet. The board B-L072Z-LRWAN ${ }^{4}$ was used as the basis, with the following key technical characteristics:

CPU - an STM32L072CZ offering an Arm ${ }^{\circledR}$ Cortex ${ }^{\circledR}$-M0+ core, with 192 Kbytes of Flash memory, 20 Kbytes of RAM and 20 Kbytes of EEPROM.

POWER - an on-board compartment on the bottom side that holds 3xAAA-sized battery.

NET - an SX1276 transceiver that features the LoRa ${ }^{\circledR}$ long-range modem including a $50 \mathrm{ohm}$ SMA RF antenna, providing ultra-long-range spread spectrum communication and high interference immunity, minimizing current consumption.

UX - four LEDs and two push-button offer simple interaction mechanisms.

IO - Arduino ${ }^{\mathrm{TM}}$ Uno V3 connectors that allow extending the boards with sensors.

COST - The cost of the board is $€ 52.70$.

The prototype device is equipped with two sensors for measuring $\mathrm{pH}$ and turbidity. Low-cost sensors were selected to reflect the budget usually available for school projects. In a usual scenario, multiple such devices will be needed for a single class to carry out the educational activities. Moreover, being handled by students and operated in environments with running water might lead to situations where certain hardware

\footnotetext{
${ }^{4}$ https://www.st.com/resource/en/user_manual/dm00329995.pdf
} 


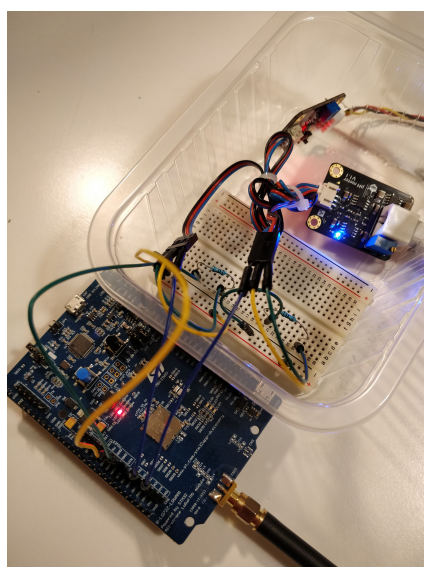

(a)

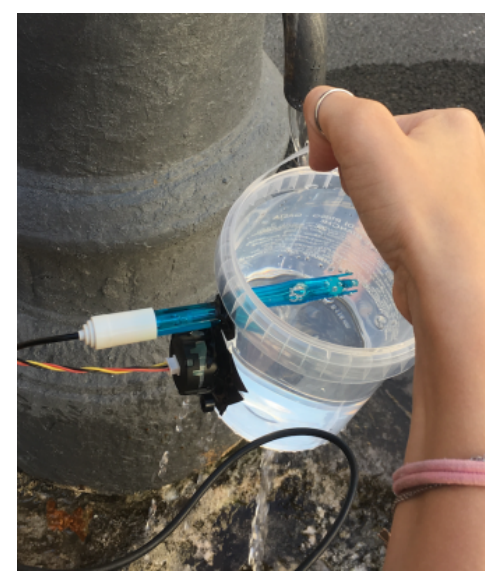

(b)

Fig. 1. Compartment for IoT device (left) and Box with sensors for sampling water (right)

elements are damaged. Note that at the time of the development of the board it was not possible to identify and acquire a low-cost temperature and dissolved oxygen sensor kit. In more details:

Water Acidity-Alkalinity - analog PH meter (SKU: SEN0161) ${ }^{5}$ operating an gain adjustment potentiometer and a $\mathrm{pH}$ electrode. The sensor achieves $\pm 0.1 \mathrm{pH}$ accuracy when measuring water at $25^{\circ} \mathrm{C}$. A standard solution whose $\mathrm{pH}$ value is 7.00 is provided to calibrate the sensor. The response time of the sensor is about $1 \mathrm{sec}$. The cost of the sensor kit is $€ 25.40$.

Water Turbidity - analog Turbidity sensor (SKU: SEN0189) ${ }^{6}$. It uses light to detect suspended particles in water by measuring the light transmittance and scattering rate, which changes with the amount of total suspended solids (TSS) in water. As the TTS increases, the liquid turbidity level increases. The response time of the sensor is about $500 \mathrm{~ms}$. The cost of the sensor kit is $€ 8.50$.

Finally, we also attach a simple GPS receiver on the board so that we can automatically identify the location of the measurement. The board, the sensors, and all other components are positioned within a simple enclosure while the two sensors are attached on the side of a small bucket to hold the water that will be sampled. Figure 1 depicts a simple implementation of the prototype using DIY components.

\subsection{Cloud Services}

The values measured by the prototype device are transmitted via the LoRa LPWAN connection to the nearest gateway provided by The Things Network ${ }^{7}$. A cloud service

\footnotetext{
${ }^{5}$ https://www.dfrobot.com/wiki/index.php/PH_meter(SKU:_SEN0161)

${ }^{6}$ https://www.dfrobot.com/wiki/index.php/Turbidity_sensor_SKU:_SEN0189

${ }^{7}$ https://www.thethingsnetwork.org/
} 
is registered to collect the data retrieved by the LoRa network and store them within a simple key-value NoSQL database. The cloud service also provides the front-end through a Node.js implementation.

\subsection{Mobile App Prototype}

A mobile application is provided as an accompanying component for the students that are on the move, engaged in the educational activities and making measurements. The mobile application acts as a diary for the students to record any remarks they might have related to the specific measurement. The main activity of the application is the display of the positions where a measurement took place on the map of the city. A secondary activity provides an overview of the values collected for a single point, the possibility to assign a label to the measurement, reposition it and add a comment. The two activities are depicted in Figure 2. The mobile application was developed for the Android environment.

\subsection{Deployment Evaluation}

The evaluation started with the examination of the sensor components and the values acquired while measuring water of various qualities. Initially, the $\mathrm{pH}$ sensor was calibrated by positioning the $\mathrm{pH}$ electrode within the standard solution provided whose $\mathrm{pH}$ value is 7.00. In the sequel, the acidity of different liquids which contain acid or base at room temperature was used to test the accuracy of the sensor. When the finalised device used to measure the quality or normal tap water, the device provided the $\mathrm{pH}$ value of 7.14 and temperature of $23^{\circ} \mathrm{C}$ and providing the turbidity value of close to the pure water $(492 \mathrm{mV})$. A series of tests were carried out at different temperatures to further test the prototype device. A lemon juice was measured at $\mathrm{pH} 2.3$, a tomato juice at 4.7 , tap water at 6.98 , distilled water at 7.02 , and soap water at 12.09 . For the case of turbidity, the highest value was recorded for distilled water $492 \mathrm{mV}$, a water mixed with a little dirt gives $374 \mathrm{mV}$ while a water mixed with a lot of dirt gave a value of $62 \mathrm{mV}$.

The next step of the evaluation focused on testing the device while carrying out measurements at outdoor. The goal was to examine the connectivity of the LoRa ${ }^{\circledR}$ long-range modem included in the main board. The device was tested in the city centre of Rome, in areas close to the Department of Computer, Control and Management Engineering (DIAG) where a LoRa ${ }^{\circledR}$ gateway device was located. In total 12 fountains were identified within a range of $700 \mathrm{~m}$ and the water coming out from these fountains were sampled 10 times. All 1200 measurements were properly received by the cloud service.

The third experiment involved a class of students aged 9 years old that were asked to use the prototype device in order to measure the water coming out from a fountain. The process of measuring water quality was explained to the students, the technical details of the particular prototype device and the individual components that constitute it was presented, and the process of taking a water sample and measuring the water as well. Moreover, it was explained to the students that the device is developed only for educational purposes and as such, it should be treated with care making sure that water does not enter the delicate hardware parts. After the presentation that lasted about 


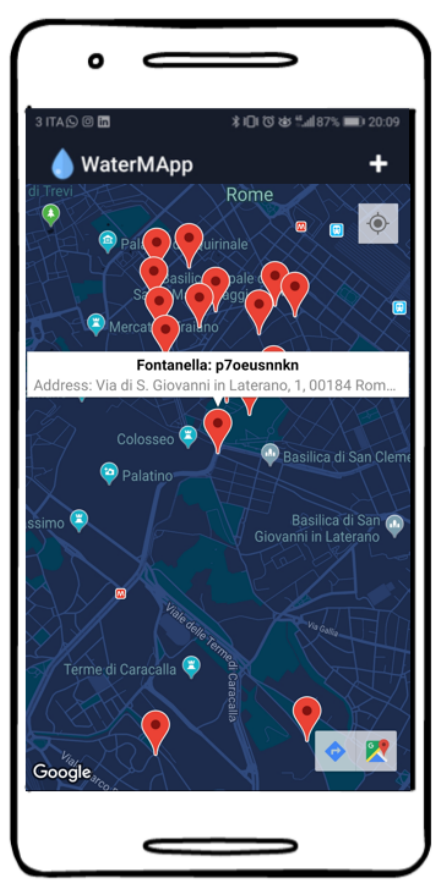

(a)

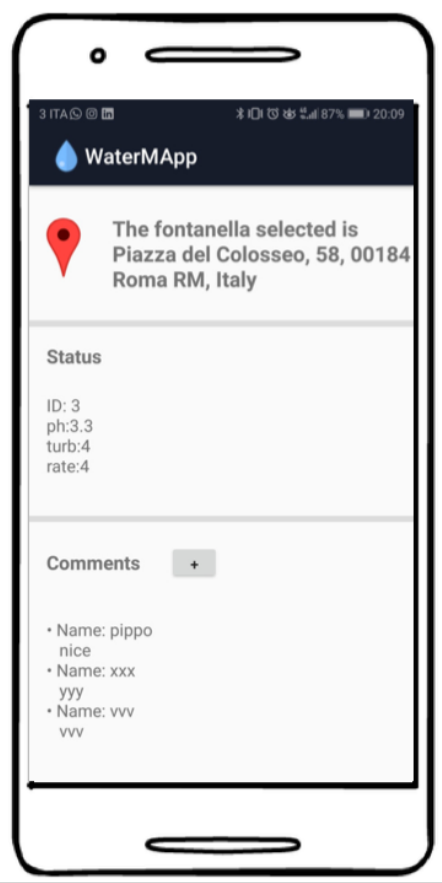

(b)

Fig. 2. Mobile application presenting the points of measurements and corresponding values

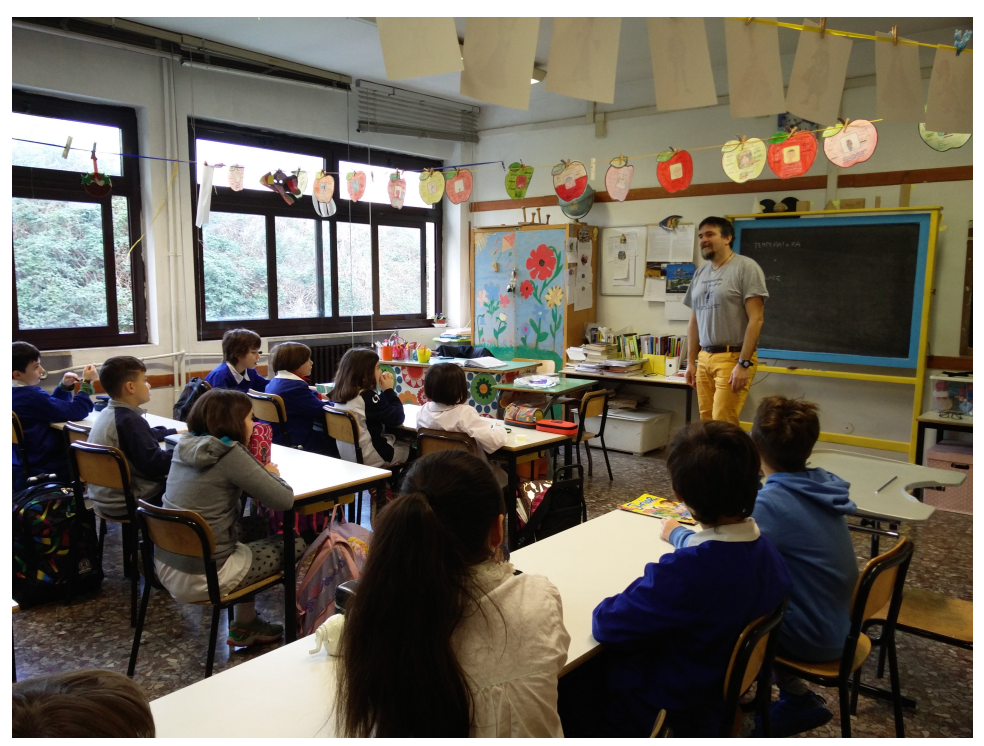

Fig. 3. Explaining the game assignment to the class 
1hour within the classroom, the students were organized in groups of 3 and each group was asked to measure the water coming out of 4 fountains positioned within $200 \mathrm{~m}$ from their school. For ensuring a reasonable quality on the measurements, the students were asked to take 3 measurements on each fountain. On average each group took about 40 minutes to carry out all the measurements. A single measurement required about 3.4 minutes, where the time was measured from the timestamps received from consecutive measurements. At the end of the experiment, all students managed to use the device properly without reporting any difficulty or damaging the device. The battery used was sufficient to sustain the operation of the device for the full day.

\section{Discussion \& Conclusions}

The main objective of sustainability education and raising awareness for environmental issues is making students aware that their actions may have a direct impact on the environment. Simple behaviour changes and interventions may have a tangible impact towards reversing water pollution. To this end, IoT technologies can support such initiatives with immediate feedback regarding the impact of their actions. This allows, on the one hand, better-informing people and enabling them to make informed decisions, and on the other to enable a different set of applications, like gamification bridging the virtual world with the real one.

The availability of actual measurements of parameters for water quality enables a number of diverse education-related applications and scenarios. For example, teachers can use collected data and analytics during class to explain phenomena related to the parameters monitored. Another example involves teachers organizing projects where students/groups monitor environmental parameters in class/home. In this direction, one major issue noted in this experiment is that IoT sensing (based on cheaper sensors and more sensitive to wear and tear) can provide values that are lacking in accuracy. The initial deployment has shown that there is often a slack between the measurements produced from the IoT sensors, that are inexpensive and are not meant as scientific measurement tools to start with. Similar differences in values of sensor readings have also been noted in the GAIA project [5], also reporting a similar approach of IoT environmental sensing used for education.

Nevertheless this slack in measuring parameters can result in important differences to the conclusions drawn; for example, during the game, water can be measured and found outside the limits of drinking values. It is therefore very important, to ensure, from the start of such games but also at regular intervals during the whole process, the appropriateness of degradation of the measurements tools used. It is important for children learning the scientific method, to learn to take correct measurements and therefore to question and grade the tools they use. Correction in degradation can be done by the school tutors themselves before they hand in the IoT sensors to their pupils, but the children need also to be notified. If one parameter is outside the acceptable margin, a warning message in the application can provide appropriate feedback and encourage to check the grading of the measurement tool, cross-check perhaps with another tool, (before drawing the wrong conclusions) and repeating the sampling actions in the same location. 
Providing appropriate feedback via the application used is essential in IoT sensing systems used in citizen science and education. When the measurements taken are not in the acceptable range or after several uses (when the worn IoT sensors may need fixing or replacement), feedback for action is needed in order to check and re-grade the IoT sensor, (and possibly provide step by step suggestions on related possible actions), so as to then repeat the measurement.

Recent technological developments allow us to extend the IoT infrastructure in order to monitor several, unforeseen before, environmental parameters, such as for example aquatic measurements. Towards this, the deployment of sensors in water is gradually introduced and promoted, with the potential aim to address more complex aquatic measurements and form appropriate underwater sensor networks for monitoring the aquatic sectors of our planet. A series of educational scenaria is proposed to this end, that utilize the collected data to further promote sustainability awareness and behavioural change. These are coupled by a gamification application, enabling the data collection as well as visualisation, and a packaged set of IoT sensors aiming specifically for water parameters. Future steps of the deployment described here is to further evaluate it, for assessing the motivation and engagement of the students for practising science and for sustaining the environment, and develop it further in the different aspects it pertains.

This research further supports that education-focused real-world IoT deployment can help to form a better understanding of our environment and promote sustainable activities, starting at a school level. By using inexpensive and easily available IoT infrastructure to measure, and then visualise and reflect on the data combinations, a more meaningful understanding of our very environment is informed, while at the same time citizen science is supported by adding to data sets.

Given the combination of technical difficulties with pedagogical goals presented in this work, it is critical to continue by a stepwise approach where advancements in the technical prototype are matched by enhancements in the gamification techniques. Detailing on the educational scenarios and how they are paired with gamification approaches are to be further researched. Likewise, it is important to carry out a high-level evaluation of the effectiveness of the proposed approach and the adoption of environmental healthy behaviour, as the ultimate goal.

\section{Acknowledgements}

We would like to thank Adriano Pimpini and Gabrio Tognozzi, students of the Sapienza University of Rome that helped in the development of the prototype system. This work has been partially supported by the EU research project "Green Awareness In Action" (GAIA), funded under contract number 696029 and the research project Designing Human-Agent Collectives for Sustainable Future Societies (C26A15TXCF) of Sapienza University of Rome. This document reflects only the authors' view and the EC and EASME are not responsible for any use that may be made of the information it contains. 


\section{References}

1. Green paper marine knowledge 2020, mapping to ocean forecasting. Technical report, Luxembourg: Publications Office of the European Union, COM (2012) 473 final, 2012. ISBN: 9789279-25350-8.

2. Whitebread, D. et al. A report on the value of childrens play with a series of policy recommendations. Technical report, Written for Toy Industries of Europe (TIE), 2012.

3. 2020 Energy Serious Game, http://www.2020energy.eu/game.

4. Orestis Akribopoulos, Ioannis Chatzigiannakis, Christos Koninis, and Evangelos Theodoridis. A web services-oriented architecture for integrating small programmable objects in the web of things. In Developments in E-systems Engineering (DESE), 2010, pages 70-75. IEEE, 2010

5. Dimitrios Amaxilatis, Orestis Akrivopoulos, Georgios Mylonas, and Ioannis Chatzigiannakis. An iot-based solution for monitoring a fleet of educational buildings focusing on energy efficiency. Sensors, 17(10):2296, 2017.

6. John Baichtal. Building Your Own Drones: A Beginners' Guide to Drones, UAVs, and ROVs. Que Publishing Company, 1st edition, 2015.

7. Dominique Brossard, Bruce Lewenstein, and Rick Bonney. Scientific knowledge and attitude change: The impact of a citizen science project. International Journal of Science Education, 27(9):1099-1121, 2005.

8. J. Burke, D. Estrin, M. Hansen, A. Parker, N. Ramanathan, S. Reddy, and M. B. Srivastava. Participatory sensing. In In: Workshop on World-Sensor-Web (WSW06): Mobile Device Centric Sensor Networks and Applications, pages 117-134, 2006.

9. Ioannis Chatzigiannakis, Henning Hasemann, Marcel Karnstedt, Oliver Kleine, Alexander Kroller, Myriam Leggieri, Dennis Pfisterer, Kay Romer, and Cuong Truong. True selfconfiguration for the iot. In Internet of Things (IOT), 2012 3rd International Conference on the, pages 9-15. IEEE, 2012.

10. Ioannis Chatzigiannakis, Alexander Kröller, and Irene Mavrommati. Indoors multi-visitors pervasive installations using mobile sensor networks. In Proceedings of the 2011 International Conference on Indoor Positioning and Navigation (IPIN '11), September 2011.

11. Ioannis Chatzigiannakis, Georgios Mylonas, Panagiotis Kokkinos, Orestis Akribopoulos, Marios Logaras, and Irene Mavrommati. Implementing multiplayer pervasive installations based on mobile sensing devices: Field experience and user evaluation from a public showcase. Journal of Systems and Software, 2011.

12. E. Chryssafidou, S. Sotiriou, P. Koulouris, M. Stratakis, A. Miliarakis, M. Barajas, M. Milrad, and D. Spikol. Developing tools that support effective mobile and game based learning: The collage platform. In In Architectures for Distributed and Complex M-Learning Systems: Applying Intelligent Technologies, 2010.

13. Charlene M Czerniak, William B Weber Jr, Alexa Sandmann, and John Ahern. A literature review of science and mathematics integration. School Science and Mathematics, 99(8):421430, 1999.

14. E.V. Dimaraki, A. Schmoelz, and P. Koulouris. Scenarios as pedagogical devices: Designing activities for game-based learning. In Proceedings of the International Conference of Education, Research and Innovation, in print.

15. Free Energy Game, PG\&E, http://www.freeenergygame.com/.

16. Jeffrey Frykholm and George Glasson. Connecting science and mathematics instruction: Pedagogical context knowledge for teachers. School Science and Mathematics, 105(3):127141, 2005.

17. Stefan Göbel, Sandro Hardy, Viktor Wendel, Florian Mehm, and Ralf Steinmetz. Serious games for health: personalized exergames. In Proceedings of the international conference on Multimedia, pages 1663-1666. ACM, 2010. 
18. Scott Heggen. Participatory sensing: Repurposing a scientific tool for stem education. interactions, 20(1):18-21, January 2013.

19. V. Kotovirta, T. Toivanen, R. Tergujeff, and M. Huttunen. Participatory sensing in environmental monitoring - experiences. In 2012 Sixth International Conference on Innovative Mobile and Internet Services in Ubiquitous Computing, pages 155-162, July 2012.

20. Edward Mallon and Patricia Beddows. The cave pearl project: developing a summersible data logger systemfor long term environmental monitoring. https://thecavepearlproject.org.

21. Koulouris P. and Dimaraki E. Engaging school communities in the design of game-based learning to foster creativity. In Proceedings of the LD-Skills: The Future of Learning Design Conference, 2012.

22. C. Petrioli, J. Potter, and R. Petroccia. Sunrise sensing, monitoring and actuating on the underwater world through a federated research infrastructure extending the future internet. In Proceedings of EMSO 2013, Rome, Italy, November, 17 2013. Demo also presented.

23. James Pierce and Eric Paulos. Materializing energy. In Proceedings of the 8th ACM Conference on Designing Interactive Systems, pages 113-122. ACM, 2010.

24. Lidia Pocero, Dimitrios Amaxilatis, Georgios Mylonas, and Ioannis Chatzigiannakis. Open source iot meter devices for smart and energy-efficient school buildings. HardwareX, 2017.

25. Siements, Power your World serious game, http://www.powermatrixgame.com.

26. Byron Reeves, James J. Cummings, James K. Scarborough, June Flora, and Dante Anderson. Leveraging the engagement of games to change energy behavior. In Waleed W. Smari and Geoffrey Charles Fox, editors, CTS, pages 354-358. IEEE, 2012.

27. Mark E Sanders. Stem, stem education, stemmania. 2008.

28. SAVE ENERGY FP7 project, http://www.ict4saveenergy.eu/.

29. Edy Hafizan Mohd Shahali, Lilia Halim, Mohamad Sattar Rasul, Kamisah Osman, and Mohd Afendi Zulkifeli. Stem learning through engineering design: Impact on middle secondary students interest towards stem. EURASIA Journal of Mathematics, Science \& Technology Education, 13(5):1189-1211, 2017.

30. W. Sun, Q. Li, and C. K. Tham. Wireless deployed and participatory sensing system for environmental monitoring. In 2014 Eleventh Annual IEEE International Conference on Sensing, Communication, and Networking (SECON), pages 158-160, June 2014. 